\title{
Single-Dose Azithromycin versus Penicillin G Benzathine for the Treatment of Early Syphilis
}

\author{
Gabriele Riedner, M.D., Ph.D., Mary Rusizoka, Dipl.Med., Jim Todd, M.Sc., \\ Leonard Maboko, M.D., Michael Hoelscher, M.D., Donan Mmbando, M.D., \\ Eleuter Samky, M.D., Eligius Lyamuya, Ph.D., David Mabey, M.D., Ph.D., \\ Heiner Grosskurth, M.D., Ph.D., and Richard Hayes, D.Sc.
}

\section{ABSTRACT}

From the London School of Hygiene and Tropical Medicine, London (G.R., J.T., D. Mabey, R.H.); the Regional Medical Office, Mbeya, Tanzania (M.R., D. Mmbando) the Mbeya Medical Research Programme, Mbeya, Tanzania (L.M.); the Department of Infectious Diseases and Tropical Medicine, Ludwig-Maximilians-University, Munich, Germany (M.H.); the Mbeya Consultant Hospital, Mbeya, Tanzania (E.S.) Muhimbili University College of Health Sciences, Dar es salaam, Tanzania (E.L.); and the Medical Research Council Programme on AIDS, Uganda Virus Research Institute, Entebbe, Uganda (H.G.). Address reprint requests to Dr. Riedner at the WHO Regional Office for the Eastern Mediterranean, Adul Razzak Al-Sanhouri St., P.O. Box 7068, Nasr City, Cairo 11371, Egypt, or at riednerg@emro.who.int.

N EnglJ Med 2005;353:1236-44. Copyright $\odot 2005$ Massachusetts Medical Society.
BACKGROUND

Pilot studies suggest that a single, 2-g oral dose of azithromycin may be an alternative to a 2.4-MU intramuscular dose of penicillin $\mathrm{G}$ benzathine in the prevention and treatment of syphilis. We evaluated the efficacy of treatment with azithromycin in a developing country.

\section{METHODS}

A total of 328 subjects, 25 with primary and 303 with high-titer (a titer of at least 1:8 on a rapid plasmin reagin [RPR] test) latent syphilis, were recruited through screening of high-risk populations in Mbeya, Tanzania, and randomly assigned to receive $2 \mathrm{~g}$ of azithromycin orally (163 subjects) or 2.4 million units of penicillin $\mathrm{G}$ benzathine intramuscularly (165 subjects). The primary outcome was treatment efficacy, with cure defined serologically (a decline in the RPR titer of at least two dilutions by nine months after treatment) and, in primary syphilis, by epithelialization of ulcers within one or two weeks.

\section{RESULTS}

The average age of participants was 27.0 years, 235 (71.6 percent) were female, and 171 (52.1 percent) were seropositive for human immunodeficiency virus. Cure rates were 97.7 percent ( 95 percent confidence interval, 94.0 to 99.4) in the azithromycin group and 95.0 percent ( 95 percent confidence interval, 90.6 to 97.8 ) in the penicillin G benzathine group ( 95 percent confidence interval for the difference, -1.7 to 7.1 percent), achieving prespecified criteria for equivalence. Cure rates were also similar three and six months after treatment in the two groups and in all subgroups. Cure rates at three months were 59.4 percent ( 95 percent confidence interval, 51.8 to 67.1) in the azithromycin group and 59.5 percent ( 95 percent confidence interval, 51.8 to 67.3 ) in the penicillin $\mathrm{G}$ benzathine group and at six months were 85.5 percent $(95$ percent confidence interval, 79.4 to 90.6 ) and 81.5 percent (95 percent confidence interval, 74.8 to 87.4 ), respectively.

\section{CONCLUSIONS}

Single-dose oral azithromycin is effective in treating syphilis and may be particularly useful in developing countries in which the use of penicillin $\mathrm{G}$ benzathine injections is problematic. However, recent reports of azithromycin-resistant Treponema pallidum in the United States indicate the importance of continued monitoring for resistance. 
SINGLE INTRAMUSCULAR INJECTION of 2.4 million units (MU) of penicillin $G$ benzathine is the recommended therapy for early syphilis. ${ }^{1}$ Major advantages of this regimen are its low cost and absence of problems related to poor adherence, whereas disadvantages include pain associated with large-volume $(4 \mathrm{ml})$, deep, intramuscular injections; the relatively high prevalence of self-reported penicillin allergy in some settings; and the need for injection equipment and medically trained personnel, which may be lacking in countries with few health resources. In addition, there are risks of transmission of blood-borne infections, such as human immunodeficiency virus (HIV), if injection equipment is reused.

The availability of an effective, well-tolerated, oral, single-dose therapy would overcome the disadvantages associated with injectable penicillin $\mathrm{G}$ benzathine. Azithromycin, a macrolide antibiotic with a long (68 hours) half-life in tissue and proven efficacy against Chlamydia trachomatis, ${ }^{2}$ Neisseria gonorrhoeae, ${ }^{3}$ and Haemophilus ducreyi, ${ }^{4}$ is a promising candidate. The results of two pilot studies suggested that the drug may be effective in preventing syphilis in persons exposed to patients with infectious cases $^{5}$ and in treating early syphilis. ${ }^{6}$ Expanding on these small pilot studies in the United States, we present the results of a randomized, controlled trial conducted in a developing country to test the hypothesis that the efficacy of a single, 2-g oral dose of azithromycin is equivalent to that of standard treatment with 2.4 MU of penicillin G benzathine for the treatment of early syphilis.

\section{METHODS}

The study protocol was approved by the ethics committees of the Tanzanian National Institute for Medical Research and the London School of Hygiene and Tropical Medicine. The study was conducted in collaboration with the Mbeya Medical Research Programme. Pfizer donated 250 doses of azithromycin but had no other involvement in the study.

\section{PARTICIPANTS}

Persons with confirmed early symptomatic syphilis (primary or secondary) or high-titer latent syphilis were recruited through screening of high-risk populations in Mbeya Region, Tanzania, including a cohort of female bar workers, patients with sexually transmitted infections attending four public clinics, and traditional-brew sellers participating in a screening and treatment intervention for sexually transmitted infections. As part of intervention activities for sexually transmitted infections, persons from these three groups were examined for clinical and serologic signs of syphilis.

Persons with presumptive primary or secondary syphilis and those with a reactive rapid plasma reagin (RPR) test (VD25, Murex Diagnostics) at the time of screening were eligible for the study. Additional eligibility criteria included an age of at least 18 years and residence in Mbeya. Exclusion criteria were pregnancy, known allergy to penicillin or macrolide antibiotics, use of antibiotics active against syphilis during the preceding six months for symptomatic cases or during the preceding two years for asymptomatic cases, and concurrent illnesses requiring treatment with antibiotics effective against syphilis.

Eligible participants were informed of the study's aims, procedures, and associated risks and provided written informed consent and a blood sample. On the following day, RPR tests were repeated and Treponema pallidum particle agglutination tests (Serodia, Fujirebio) were performed on blood samples at the research laboratory of the Mbeya Medical Research Programme in Mbeya. As planned before the start of the study, if RPR titers were found to be 1:4 or less or the T. pallidum particle agglutination test was nonreactive, participants were retrospectively excluded from the trial unless primary syphilis was confirmed by the finding of T. pallidum in ulcer exudates on polymerase chain reaction (PCR). ${ }^{7}$

Among eligible participants, HIV serologic status was determined through a dual enzyme-linked immunosorbent assay (Determine HIV 1/2, Abbott, and Enzygnost HIV 1/2 plus, Behring) of samples. All consenting subjects were offered counseling before and after undergoing tests for HIV. Eligible HIV-infected participants received cotrimoxazole prophylaxis and were referred to existing support services in Mbeya, through which further treatment of opportunistic infections was available. The study was conducted between September 2000 and September 2003.

\section{DEFINITIONS}

Primary and secondary syphilis were defined by the presence of genital ulcers or condylomata lata, respectively, confirmed to be positive for T. pallidum on PCR. High-titer latent syphilis was defined on the basis of a reactive T. pallidum particle agglutination test and an RPR titer of at least 1:8 in the ab- 
sence of ulcers or condylomata lata confirmed to be positive for T. pallidum on PCR.

The primary outcome was serologic cure, defined as a decrease in the RPR titer by at least two dilutions before or at the nine-month follow-up examination, with the titer at the time of treatment used as the baseline. In the case of primary and secondary syphilis, complete resolution or improvement (epithelialization in progress) of lesions within one or two weeks after treatment was also required. Secondary outcomes were cure rates three and six months after treatment and cure rates according to age, sex, stage of syphilis, and RPR titer at treatment. Treatment was considered to have failed if the RPR titer increased by two or more dilutions as compared with the baseline titer before cure or if the titer had not declined by at least two dilutions within nine months. Participants discontinued their participation in the study once cured. Thus, increases in titers after cure, possibly indicating relapse or reinfection, would not be identified. Before discharging participants from the study, we determined all titers in parallel using stored specimens, to avoid any error resulting from testing of unpaired specimens.

\section{RANDOMIZATION AND FOLLOW-UP}

A random allocation schedule, stratified according to study group, was generated centrally with the use of blocked randomization, random permuted blocks of four, and a 1:1 allocation ratio. The allocation was concealed from investigators through the use of sequentially numbered, sealed envelopes.

Participants with primary syphilis were reexamined one or two weeks after treatment to assess whether syphilitic lesions had resolved. All participants were reassessed every three months for up to nine months until they were cured. At each followup examination, participants reported any sexual exposure between visits and were clinically evaluated for persistent or recurrent syphilis. A 5-ml blood sample was obtained for serologic analysis for syphilis. Laboratory technicians were unaware of participants' treatment allocation, treatment response, and previous RPR test results at all times. All RPR tests were carried out in duplicate by two independent technicians, and tests were repeated in cases of discrepant results.

\section{STATISTICAL ANALYSIS}

In this equivalence trial, we estimated that at least 133 participants were required in each group for the study to have a statistical power of 80 percent to exclude the possibility that the absolute efficacy of azithromycin was at least 7.5 percent less than that of penicillin (with a one-sided $\mathrm{P}<0.05$ ), assuming that the true efficacy of each agent was equivalent at 95 percent and that approximately 30 percent of participants would be lost to follow-up.

A primary analysis was carried out to estimate cure rates at or before the three-, six-, and ninemonth follow-up visit with the use of Kaplan-Meier methods. Data on participants who were lost to follow-up before being cured were censored on the date of the last follow-up visit. Differences between groups in the cure rates at nine months were assessed with the use of an approximate z-test after complementary log-log transformation, whereas overall differences in the time to cure between groups were assessed with the use of the log-rank test. Since participants did not always attend their scheduled appointments on time, a secondary analysis was performed based on exact times from treatment to follow-up visits with the use of the KaplanMeier method. In this analysis, cure was assumed to have occurred at the midpoint between two successive examinations. On the basis of the secondary analysis (exact follow-up times), significant determinants of cure were included in a Cox regression model to examine their joint effect on cure rates. Two-sided tests were used throughout. All statistical analyses were carried out with the use of Stata software (version 8).

\section{RESULTS}

From September 2000 to March 2003, 6308 eligibility assessments were performed. Of 628 persons recruited on site, 300 were retrospectively found to be ineligible on the basis of serologic results for syphilis at the reference laboratory (Fig. 1). Thus, 328 persons fulfilled the inclusion criteria: $65 \mathrm{fe}$ male bar workers, 149 attendees of a sexually transmitted infection clinic, and 114 traditional-brew sellers. Of these 328 participants, 165 (50.3 percent) received penicillin $\mathrm{G}$ benzathine and 163 (49.7 percent) azithromycin (Zithromax, Pfizer).

The average age of participants was 27.0 years (range, 15 to 60), 235 (71.6 percent) were female, and 171 (52.1 percent) were seropositive for HIV (Table 1). Twelve participants were younger than 18 years of age, having misreported their age at the time of recruitment, but were included in the analysis. Primary syphilis was confirmed by PCR in 25 


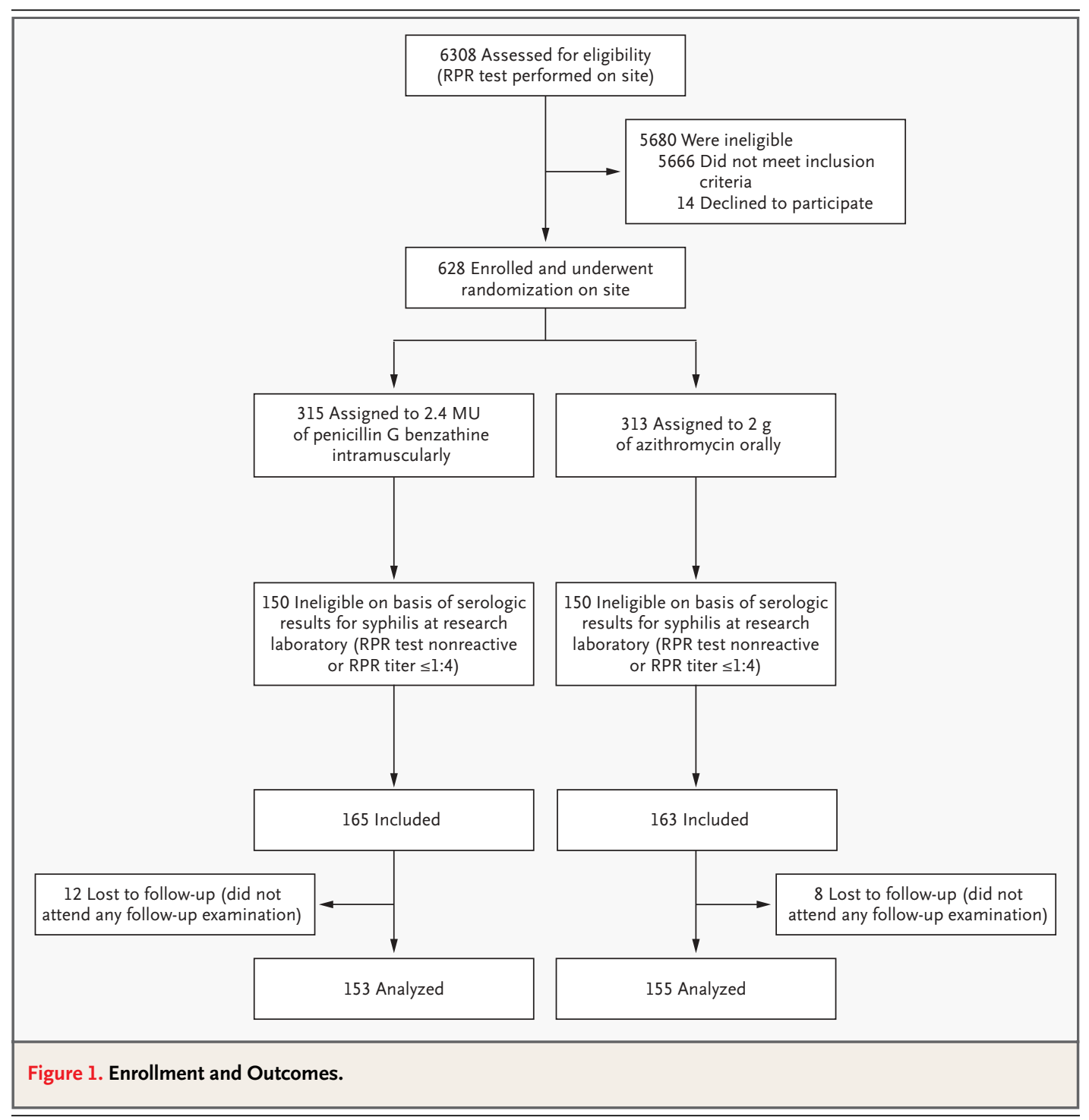

(7.6 percent) participants. There were no PCR-confirmed cases of secondary syphilis. The RPR titer was no more than 1:32 in 217 (66.2 percent) participants and at least 1:64 in 111 (33.8 percent). The two treatment groups were generally similar with regard to sociodemographic, behavioral, and biologic characteristics (Table 1 ).

Of the 328 participants, 31 (9.5 percent) were lost to follow-up, including 20 (6.1 percent) who did not attend any follow-up visits and who were excluded from the analysis of cure rates. An additional 11 participants ( 3.4 percent), 6 in the azithromycin group and 5 in the penicillin $\mathrm{G}$ benzathine group, were lost after the first follow-up visit. Four of these (two in each group) were excluded after having been re-treated for presumptive symptomatic reinfection or relapse (ulcers or rash). PCR testing of these lesions was not performed, but serologic analysis was not suggestive of relapse or reinfection, since RPR titers showed no increase.

Among those treated with azithromycin, 140 participants were interviewed about adverse events at the first follow-up visit. A total of 12 reported nausea (8.6 percent), 6 stomach pain ( 4.3 percent), 1 diarrhea ( 0.7 percent), and 1 vomiting ( 0.7 percent).

Among the 24 of 25 participants with confirmed primary syphilis who were examined one or two weeks after treatment, ulcers had resolved in 15 ( 62.5 percent) and were in the process of healing in 9 (37.5 percent). There was no significant differ- 


\begin{tabular}{|c|c|c|c|}
\hline Characteristic & $\begin{array}{c}\text { Penicillin G } \\
\text { Benzathine } \\
(\mathrm{N}=165)\end{array}$ & $\begin{array}{l}\text { Azithromycin } \\
(N=163)\end{array}$ & $\begin{array}{c}\text { Total } \\
(\mathrm{N}=328)\end{array}$ \\
\hline & \multicolumn{3}{|c|}{ number (percent) } \\
\hline \multicolumn{4}{|l|}{ Study group } \\
\hline Female bar workers & $33(20.0)$ & $32(19.6)$ & $65(19.8)$ \\
\hline $\begin{array}{l}\text { Patients attending a sexually } \\
\text { transmitted infection clinic }\end{array}$ & $71(43.0)$ & $78(47.9)$ & $149(45.4)$ \\
\hline Traditional-brew sellers & $61(37.0)$ & $53(32.5)$ & $114(34.8)$ \\
\hline \multicolumn{4}{|l|}{ Sex } \\
\hline Male & $41(24.8)$ & $52(31.9)$ & $93(28.4)$ \\
\hline Female & $124(75.2)$ & $111(68.1)$ & $235(71.6)$ \\
\hline \multicolumn{4}{|l|}{ Age } \\
\hline $15-24 \mathrm{yr}$ & $77(46.7)$ & $69(42.3)$ & $146(44.5)$ \\
\hline$\geq 25 \mathrm{yr}$ & $88(53.3)$ & $94(57.7)$ & $182(55.5)$ \\
\hline \multicolumn{4}{|l|}{ No. of sexual partners in past $3 \mathrm{mo*}^{*}$} \\
\hline$\leq 1$ & $121(75.2)$ & $110(68.8)$ & $231(72.0)$ \\
\hline$\geq 2$ & $40(24.8)$ & $50(31.2)$ & $90(28.0)$ \\
\hline \multicolumn{4}{|l|}{ HIV status } \\
\hline Negative & $78(47.3)$ & $79(48.5)$ & $157(47.9)$ \\
\hline Positive & $87(52.7)$ & $84(51.5)$ & $171(52.1)$ \\
\hline \multicolumn{4}{|l|}{ Syphilis stage } \\
\hline Primary & $14(8.5)$ & $11(6.7)$ & $25(7.6)$ \\
\hline Latent & $151(91.5)$ & $152(93.3)$ & $303(92.4)$ \\
\hline \multicolumn{4}{|l|}{ RPR titer at treatment } \\
\hline$\leq 1: 32$ & $110(66.7)$ & $107(65.6)$ & $217(66.2)$ \\
\hline$\geq 1: 64$ & $55(33.3)$ & $56(34.4)$ & $111(33.8)$ \\
\hline
\end{tabular}

* Values were missing for four subjects in the penicillin group and three in the azithromycin group. cating that the possibility that a cure rate that was 7.5 percentage points lower for azithromycin than for penicillin $\mathrm{G}$ benzathine could be confidently excluded $(z=3.09, \mathrm{P}<0.002)$ (Table 2$)$. Cure rates at the three-month follow-up visit were 59.4 percent (95 percent confidence interval, 51.8 to 67.1) in the azithromycin group and 59.5 percent $(95$ percent confidence interval, 51.8 to 67.3 ) in the penicillin $\mathrm{G}$ benzathine group, and cure rates at the six-month follow-up were 85.5 percent $(95$ percent confidence interval, 79.4 to 90.6 ) and 81.5 percent (95 percent confidence interval, 74.8 to 87.4 ), respectively (Table 2 and Fig. 2). Consistent findings were obtained in subgroup analyses based on study group, stage of syphilis, baseline RPR titer, and HIV status (Table 2) and age and sex (data not shown), with azithromycin showing equal or greater efficacy in all subgroups examined.

Since there was substantial variation between participants in the timing of follow-up visits, with ranges from 32 to 133 days for the 3-month visit, 130 to 229 days for the 6-month visit, and 207 to 304 days for the 9-month visit, we conducted a secondary analysis that was based on the exact intervals between treatment and follow-up examinations. The findings were similar, with a cure rate at nine months of 98.3 percent ( 95 percent confidence interval, 94.5 to 99.7) in the azithromycin group and 96.5 percent ( 95 percent confidence interval, 92.0 to 98.8) in the penicillin $\mathrm{G}$ benzathine group.

Subgroup analyses were also used to identify sociodemographic and biologic determinants of cure rates in the two groups. Neither age nor sex was significantly associated with cure rates. Survival curves for the time to cure on the basis of the secondary analysis in selected subgroups are shown in Figure 3. The time between treatment and cure was shorter among participants with primary syphilis than among those with latent cases (Fig. 3B) and among participants with RPR titers of at least 1:64 than among those with lower titers (Fig. 3C), although the cure rates by nine months were high in all subgroups. HIV status had no consistent effect on cure rates. At nine months, the cure rate was 94.7 percent ( 95 percent confidence interval, 90.4 to 97.5 ) among HIV-positive participants and 98.4 percent (95 percent confidence interval, 94.8 to 99.7) among HIV-negative participants ( $\mathrm{P}>0.5$ ) (Fig. 3D).

In a Cox regression model including baseline RPR titer and syphilis stage, the adjusted hazard ratio (based on time to cure) for treatment with azithromycin, as compared with treatment with penicil- 


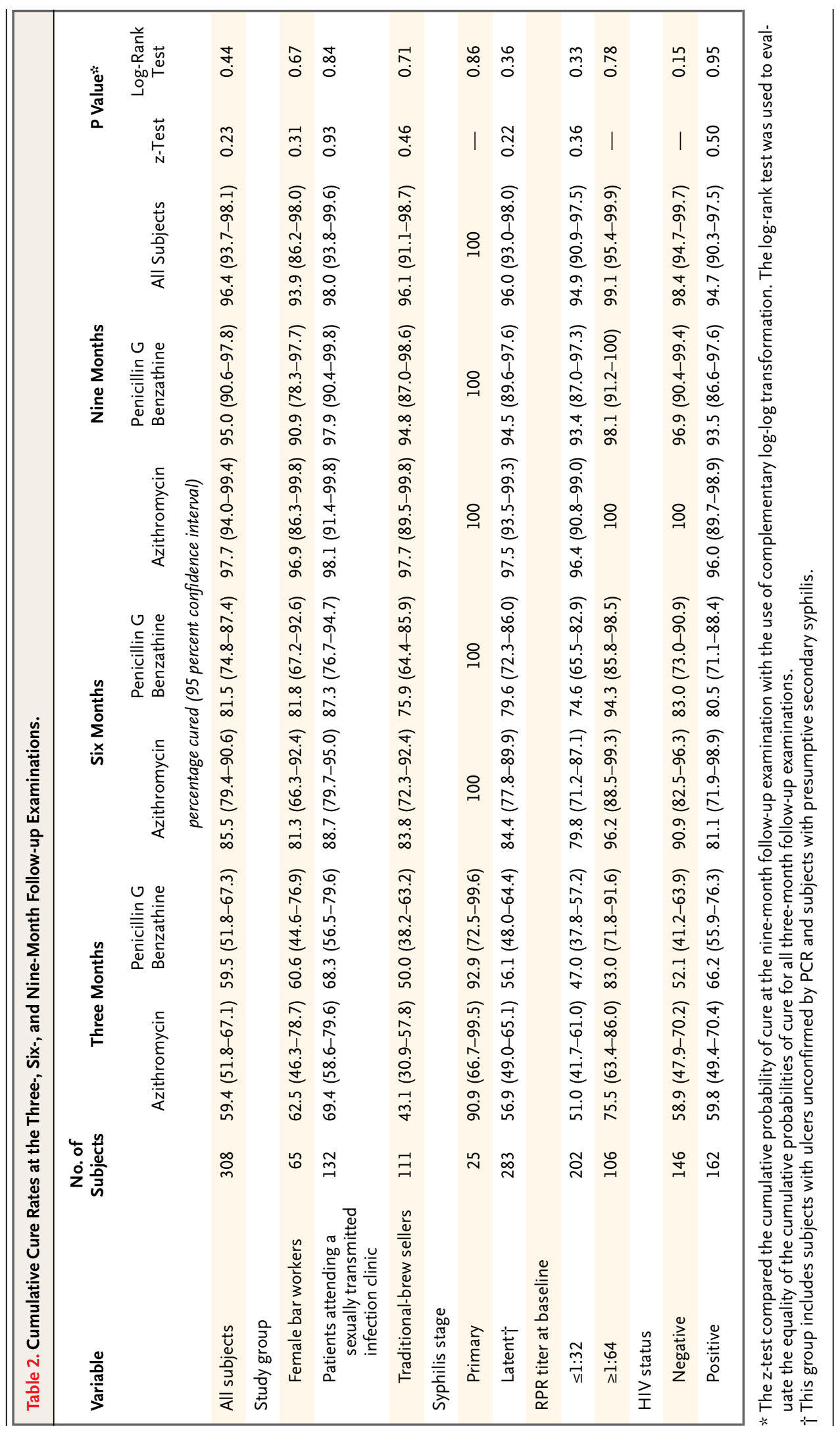

N ENGL J MED 353;12 WWW.NEJM.ORG SEPTEMBER 22, 2005 


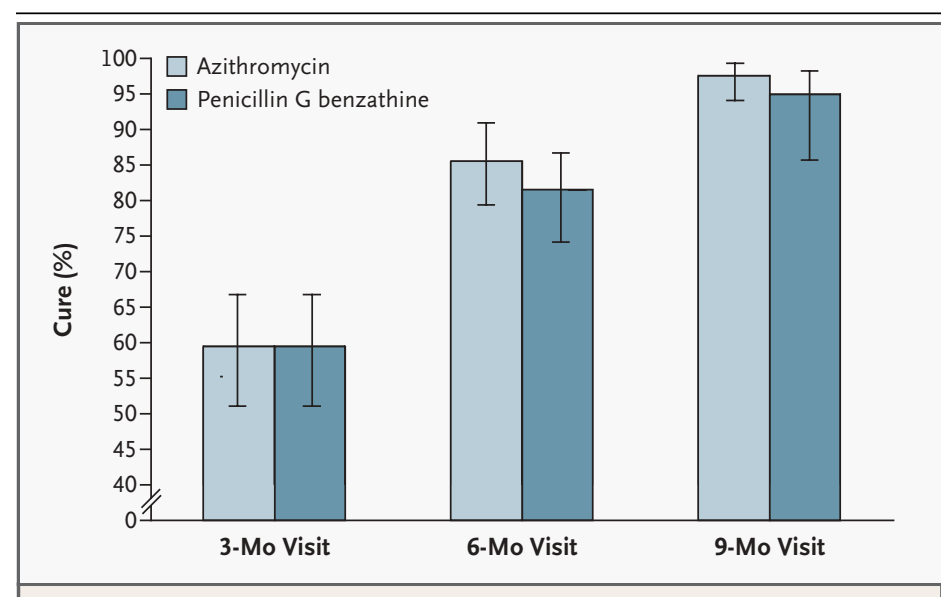

Figure 2. Mean Cure Rates.

lin $\mathrm{G}$ benzathine, was 1.15 ( 95 percent confidence interval, 0.85 to 1.36); that for an RPR titer of at least 1:64, as compared with a titer of no more than 1:32, was 2.12 ( 95 percent confidence interval, 1.66 to 2.72); that for primary syphilis, as compared with latent syphilis, was 1.98 ( 95 percent confidence interval, 1.30 to 3.01); and that for HIV-positive status, as compared with HIV-negative status, was 0.89 (95 percent confidence interval, 0.71 to 1.13 ).

\section{I SCUSS I O N}

The treatment efficacy of the two regimens was equivalent at 97.7 percent ( 95 percent confidence interval, 94.0 to 99.4) for azithromycin and 95.0 percent (95 percent confidence interval, 90.6 to 97.8 ) for penicillin $\mathrm{G}$ benzathine. The 95 percent confidence interval for the difference in cure rates ranged from -1.7 percent to 7.1 percent in favor of azithromycin, indicating that a cure rate for azithromycin that was 7.5 percentage points lower than that for penicillin $G$ benzathine was very unlikely. In addition, the cure rates were similar in the two groups throughout the follow-up period and in subgroups defined according to sociodemographic and biologic characteristics. Within the limitations of current serologic means of identifying cure, we clearly demonstrated that $2 \mathrm{~g}$ of azithromycin is as effective as 2.4 MU of penicillin G benzathine in treating early syphilis in this study population.

It is reassuring that no treatment failure was observed among participants with confirmed primary syphilis. However, a recent report from the Centers for Disease Control and Prevention of well-docu- mented treatment failure in three patients treated with $2 \mathrm{~g}$ of azithromycin for primary syphilis and the detection of a strain of T. pallidum with functional azithromycin resistance among selected groups with high-risk sexual behavior in the United States and Ireland have aroused concern about the efficacy of this regimen. ${ }^{8,9}$ In Mbeya, all cases of primary syphilis were clinically cured by the three-month follow-up visit and showed evidence of serologic cure by the six-month follow-up visit. It is unfortunate that PCR was not performed on specimens from the nine participants (four treated with azithromycin and five with penicillin) whose primary lesions had not completely resolved after one or two weeks, since biologic treatment failures could then have been verified or ruled out. Our findings do not suggest that azithromycin-resistant strains of T. pallidum are widespread in Tanzania at this time, but the observations from the United States and Ireland emphasize the importance of ongoing monitoring for drug resistance.

Among the 140 participants who were treated with azithromycin and interviewed, 11.4 percent reported mild-to-moderate side effects that were predominantly gastrointestinal. It appears that with the high, 2-g dose of azithromycin, the frequency of side effects was no higher than that reported previously for lower doses. ${ }^{10}$

Previous studies assessing the response of patients with early syphilis to one 2.4-MU dose of penicillin $\mathrm{G}$ benzathine have raised doubts concerning the effectiveness of this regimen in HIV-infected patients. ${ }^{11}$ Our findings do not confirm these observations. Although there were too few participants with primary syphilis for us to detect small differences in efficacy, the cure rate was 100 percent in the small number of HIV-positive participants with primary syphilis. The cure rate among all HIV-positive participants with latent syphilis was, at 92.1 percent, slightly lower than the 98.6 percent response among participants without HIV infection, but this difference was not statistically significant. It is known that $T$. pallidum frequently invades the central nervous system in early syphilis and that it may persist there for months after treatment without causing early signs or symptoms of neurosyphilis even if nontreponemal test titers indicate the occurrence of a serologic cure, ${ }^{11}$ and there is concern that HIV-positive patients may be at increased risk for neurosyphilis. ${ }^{12}$ Since we did not obtain cerebrospinal fluid for diagnostic tests, we cannot definitively rule out this possibility. 


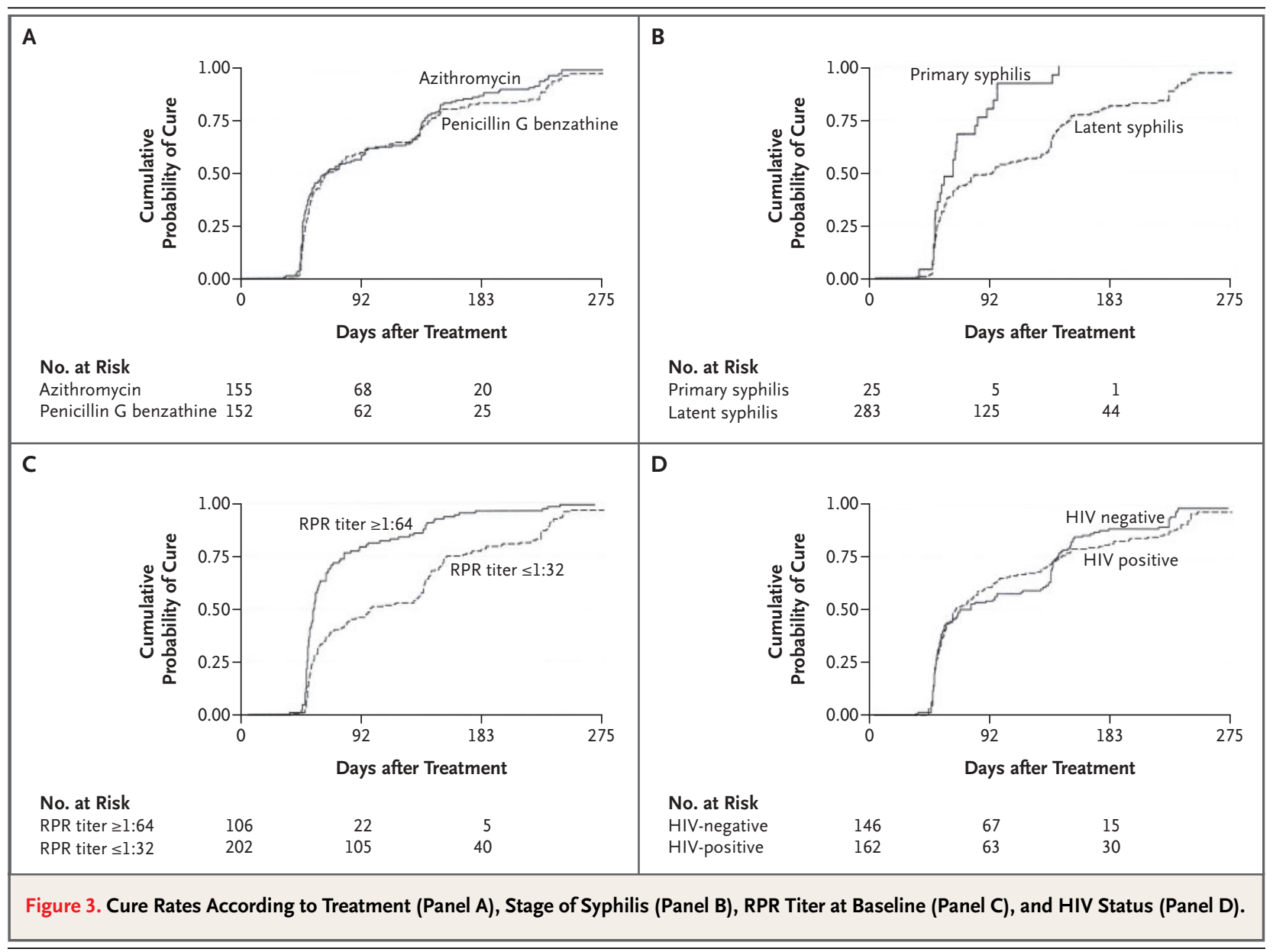

Because it was difficult to obtain reliable medical histories, we defined early latent syphilis exclusively on the basis of an RPR titer of at least 1:8 in the absence of syphilitic lesions confirmed to be positive for T. pallidum by PCR. Although RPR titers are assumed to fall during the course of latent infection, the use of this definition will have introduced some degree of (nondifferential) misclassification, with the inclusion of some participants with late latent cases. Our results are therefore applicable to a mixed population of persons with early and latent cases, making them highly relevant to syphilis-control strategies in developing countries in which, for example, the duration of infection is usually unknown in women being screened for syphilis during antenatal care.

A major problem affecting research on syphilis treatment is the imprecision of the definition of serologic cure. Such imprecision may have led us either to overestimate or to underestimate the true rates of cure. Since laboratory technicians were un- aware of participants' treatment assignments, however, this should not have biased the comparison of cure rates in the two groups.

Finally, we could not identify late relapses after serologic cure, since we essentially did not collect information on the clinical and serologic history of participants after they had been cured. The exception was female bar workers, who were followed as part of other research activities. Relapse is assumed to be highly unlikely if a satisfactory decline in the nontreponemal test titer occurs in the absence of symptoms and signs of disease and is maintained for 12 months. ${ }^{13}$ Since participants were not followed once they were cured, however, some may have had an unrecorded relapse after serologic cure. In 8 of 65 bar workers participating in the trial, increases in the RPR titer of at least two dilutions were detected after they were considered serologically cured. One had an increase in the RPR titer 6 months after cure, and the rest had increases 9 to 15 months after cure. Four of these increases in RPR titer oc- 
curred among the 15 HIV-negative bar workers and 4 among the 50 HIV-positive bar workers. It is likely that some or all of the increases in RPR titer in this highly exposed group were due to reinfection. If one assumes that all were due to relapse, the proportions with a relapse more than nine months after treatment were 12.5 percent ( 95 percent confidence interval, 3.5 to 29.0) in the azithromycin group and 12.1 percent ( 95 percent confidence interval, 3.4 to 28.2 ) in the penicillin $G$ benzathine group. On the basis of these limited data, therefore, there is no indication that the relapse rate was higher in either group. However, a definitive answer to this question would require long-term follow-up of a lower-risk study population in which reinfections are less likely.

In conclusion, this randomized, controlled trial has provided clear evidence that a single, 2-g dose of azithromycin is as effective as a 2.4-MU dose of penicillin $\mathrm{G}$ benzathine for the treatment of early syphilis. Although continued monitoring for azithromycin resistance will be essential, given the logistical advantages conferred by this oral treatment, particularly in resource-poor settings in developing countries, and the efficacy of azithromycin in treating other common sexually transmitted infections that may be present with syphilis as mixed infections, our findings support the wider use of this alternative regimen in syphilis-control programs.

Supported by a Research Training Fellowship (to Dr. Riedner) from the Wellcome Trust, United Kingdom, and a grant from the European Commission (DG XII, INCO-DC).

We are indebted to the women and men who participated in the trial, the research team of the Mbeya Syphilis Studies and of the Mbeya Medical Research Programme, the medical officer in charge of Mbeya municipality, and the personnel of the Igawilo, K'mpaka, Ruanda, and Mbeya Consultant Hospital clinics and laboratories for making this study possible; to Fred Mhalu and Frank von Sonnenburg for their contributions to the initial planning of the study; to Oliver Hoffmann, Karl-Heinz Herbinger, Britta Dechamps, Gudrun Schoen, and Frowin Nichombe for their active support in Mbeya; and to Eddy van Dyck, Tania Crucitti, and Ian MacLean for their contributions to laboratory testing.
REFERENCES

1. Parkes R, Renton A, Meheus A, LaukammJosten U. Review of current evidence and comparison of guidelines for effective syphilis treatment in Europe. Int J STD AIDS 2004;15:73-88.

2. Martin DH, Mroczkowski TF, Dalu ZA, et al. A controlled trial of a single dose of azithromycin for the treatment of chlamyd ial urethritis and cervicitis. N Engl J Med 1992;327:921-5.

3. Handsfield HH, Dalu ZA, Martin DH Douglas JM Jr, McCarty JM, Schlossberg D. Multicenter trial of single-dose azithromy cin vs. ceftriaxone in the treatment of uncomplicated gonorrhea. Sex Transm Dis 1994 21:107-11.

4. Martin DH, Sargent SJ, Wendel GD Jr, McCormack WM, Spier NA, Johnson RB. Comparison of azithromycin and ceftriaxone for the treatment of chancroid. Clin Infect Dis 1995;21:409-14.
5. Hook EW III, Stephens J, Ennis DM. Azithromycin compared with penicillin G benzathine for treatment of incubating syphilis. Ann Intern Med 1999;131:434-7.

6. Hook EW III, Martin DH, Stephens J, Smith BS, Smith K. A randomized, comparative pilot study of azithromycin versus penicillin G benzathine for treatment of early syphilis. Sex Transm Dis 2002;29:486-90.

7. Orle KA, Gates CA, Martin DH, Body BA, Weiss JB. Simultaneous PCR detection of Haemophilus ducreyi, Treponema pallidum, and herpes simplex virus types 1 and 2 from genital ulcers. J Clin Microbiol 1996; 34:49-54.

8. Azithromycin treatment failures in syphilis infections - San Francisco, California, 2002-2003. MMWR Morb Mortal Wkly Rep 2004;53:197-8.

9. Lukehart SA, Godornes C, Molini BJ, et al. Macrolide resistance in Treponema palli- dum in the United States and Ireland. N Engl J Med 2004;351:154-8.

10. Hopkins S. Clinical toleration and safety of azithromycin. Am J Med 1991;91:40S$45 \mathrm{~S}$.

11. Rolfs RT, Joesoef MR, Hendershot EF, et al. A randomized trial of enhanced therapy for early syphilis in patients with and without human immunodeficiency virus infection. N Engl J Med 1997;337:307-14

12. Musher DM, Hamill RJ, Baughn RE. Effect of human immunodeficiency virus (HIV) infection on the course of syphilis and on the response to treatment. Ann Intern Med 1990;113:872-81.

13. Hart G. Syphilis tests in diagnostic and therapeutic decision making. Ann Intern Med 1986;104:368-76.

Copyright (c) 2005 Massachusetts Medical Society. 